Faculty \& Staff Scholarship

2006

\title{
A Tabu Search Heuristic for the Dynamic Space Allocation Problem
}

Alan McKendall

Juan Jaramillo

Follow this and additional works at: https://researchrepository.wvu.edu/faculty_publications

Part of the Operations Research, Systems Engineering and Industrial Engineering Commons 


\title{
A tabu search heuristic for the dynamic space allocation problem
}

\author{
Alan R. McKendall Jr.*, J.R. Jaramillo \\ Department of Industrial \& Management Systems Engineering, West Virginia University, 325A Mineral Resources Building, \\ Morgantown, $W V$ 26506, USA
}

Available online 25 September 2004

\begin{abstract}
The dynamic space allocation problem (DSAP) presented here is a relatively new problem in the literature. It looks at the optimization of space/resource assignments during the implementation of project activities. More specifically, the DSAP assigns project activities and their required resources to workspaces and idle resources to storage spaces with respect to minimizing the sum of the distances the resources travel. In this paper, construction algorithms and a tabu search heuristic are presented for the DSAP, and a set of test problems taken from the literature is used to test the performances of the heuristics. The results show that the proposed tabu search heuristic clearly outperforms the techniques (i.e., simulated annealing heuristics) presented in the literature with respect to solution quality and computation time.
\end{abstract}

(C) 2004 Elsevier Ltd. All rights reserved.

Keywords: Dynamic space allocation problem; Tabu search; Clustering algorithms; Randomized storage policy

\section{Introduction}

The dynamic space allocation problem (DSAP) presented here is a relatively new problem in the literature. It was first defined by McKendall et al. [1]. The problem was defined as the task of assigning maintenance activities and their required resources to workspaces and idle resources to storage spaces such that the total distance the resources travel throughout the duration of an outage at an electric power plant is minimized. The DSAP defined in this paper looks at the optimization of resource/space assignments during the implementation of project activities such that the total distance traveled by the resources required to perform the activities of a project is minimized. In other words, when project activities requiring resources

\footnotetext{
* Corresponding author. Fax: +1-304-293-4970.

E-mail address: armckendall@mail.wvu.edu (A.R. McKendall).
} 
are scheduled such that the locations of the resources need to be determined with respect to minimizing the total distance the resources travel throughout the duration of the project, this problem is defined as the DSAP. Besides managing resources during electric power plant outages (McKendall et al. [1]), the DSAP has potential applications in managing resources during the overhaul of complex equipment (Gharbi et al. [2]), construction of buildings (Zouein and Tommelein [3]), underground mining and in any situation in which the movement of resources is difficult, expensive, or congestion is undesirable. In all of these examples or projects, space (work/storage) is a scarce resource used to perform activities and to store idle resources. Also, material handling equipment such as cranes and lift trucks are used to move resources to/from work/storage spaces. Therefore, efficiently allocating activities, tools, spare parts, construction materials, and/or mining equipment to work/storage spaces during the implementation of project activities is necessary to reduce congestion and work crew interference as well as to reduce material handling costs.

The DSAP is closely related to the dynamic facility layout problem (DFLP) presented by Rosenblatt [4], which is modeled as a general quadratic assignment problem (QAP). The QAP is defined as the allocation of $n$ facilities to $n$ locations while minimizing the total cost. Total cost may include material handling cost and allocation cost of each facility to a specific location (Koopmans and Beckmann [5]). However, the DFLP minimizes the material handling cost and the relocation cost when a facility is redesigned multiple times during the planning horizon (Balakrishnan and Cheng [6]). The DFLP can be viewed as a succession of QAPs. In each period of the DFLP, a QAP is required to be solved. That is, the QAP is a DFLP with only one period in the planning horizon. Since solving the DFLP as a sequence of QAPs can produce layouts with low material handling costs and extremely high rearrangement costs, the objective is to produce a set of layouts with respect to minimizing the sum of the material handling and rearrangement costs. Therefore, the DFLP is more difficult than the QAP, which has been categorized as an NP hard problem (Sahni and Gonzalez [7]).

The DSAP is similar to the DFLP. The DSAP assigns resources (both required and idle resources) to locations, while the DFLP assigns facilities or departments to locations. The main differences between the DSAP and the DFLP are related to the restrictions on the assignment of the locations. The DSAP assigns idle resources to storage locations (storages spaces) and activities (i.e., required resources) to work locations (workspaces). In contrast, the DFLP allocate facilities/departments to locations. In addition, the DSAP may assign more than one resource to each location (considering the capacity of the locations), while the DFLP assigns only one facility/department to each location. Similarly, the DSAP and the DFLP look for a series of layouts (i.e., one for each period considered in the planning horizon), which minimizes the total cost of the layout plans. Cost in the DSAP is the total distance traveled by the resources during the planning horizon, and cost in the DFLP is the material handling cost (i.e., sum of the distance materials travel times the flow of materials between two departments) plus rearrangement cost. Therefore, the DSAP is a generalization of both the QAP and the DFLP. Since the QAP is an NP hard problem and the DSAP is a generalization of the QAP, the DSAP is computationally difficult to solve optimally in reasonable time. In light of the literature review, there is only one paper that addresses the DSAP defined in this paper, which is the paper presented by McKendall et al. [1]. The authors presented two formulations (0/1 integer nonlinear and 0/1 integer linear programs) and two simulated annealing heuristics for the DSAP, as it relates to assigning maintenance activities to workspaces and resources to work/storage spaces during planned outages at electric power plants.

The purpose of this paper is to present a tabu search heuristic as well as several construction algorithms for the DSAP. The construction algorithms are used to generate initial solutions for the tabu search heuristic. In Section 2, the definition, assumptions, and a small problem instance are given for the DSAP. 
Then a tabu search heuristic for the DSAP is presented in Section 3, and several constructions algorithms for generating initial solutions for the tabu search heuristic are presented in Section 4. In Section 5, the computational results of the proposed techniques on a set of test problems taken from the literature are given. Finally, Section 6 provides conclusions and future research directions.

\section{The dynamic space allocation problem}

When performing project activities where space is limited, the locations of the activities and their required resources need to be determined such that the total distance the resources travel throughout the duration of the project is minimized. During certain time periods, some resources are required to perform activities, and others are idle. When a resource is idle (not required by an activity), it is assigned to a storage location. Therefore, the DSAP assigns activities and their required resources to workspaces and idle resources to storage spaces with respect to minimizing the total distance resources travel throughout the duration of a project. The inputs of the problem are: the schedule of the activities; the lists of resources required to perform each activity; the locations in which activities can be performed (workspaces); the locations in which idle resources can be stored (storage spaces); the capacities of the storage spaces; and the distances between locations. The outputs of the problem are: the assignments of activities (and their required resources) to workspaces; the assignments of idle resources to storage spaces; and the total distance traveled by the resources during project execution. For an example of a small DSAP instance with five periods, five activities, eight resources, and six locations (three workspaces and three storage spaces), see Table 1 for the schedule of activities. For instance, activity 3 is performed in periods 1, 2, and 3 and requires resources 1 and 6 . Also, resource 4 is idle in period 1 . The layout of the facility is given in Fig. 1 where locations 1, 2, and 3 are the workspaces, and locations 4, 5, and 6 are the storage spaces. The rectilinear distance measure is used to obtain the distances between locations and is given in Table 2. For example, the distance between locations 1 and 6 is 3 distance units. Also, the maximum capacity of each storage space is 3 resources. A solution of the DSAP is given in Fig. 2, which has a cost

Table 1

Schedule of activities for a small DSAP instance

\begin{tabular}{llll}
\hline Period & Activity & Resources & Idle resources \\
\hline 1 & 1 & $5,7,8$ & 4 \\
& 2 & 2,3 & 1,6 \\
& 3 & 1,6 & $2,3,4,5,7,8$ \\
2 & 3 & 1,6 & $2,3,5,8$ \\
3 & 3 & 4,7 & $1,3,6,8$ \\
4 & 4 & 4,7 & 2,5 \\
5 & 4 & 4,7 & $1,2,3,5,6,8$ \\
\hline
\end{tabular}




\begin{tabular}{|c|c|c|}
\hline $\begin{array}{c}\text { Location 1 } \\
\text { (Workspace) }\end{array}$ & $\begin{array}{c}\text { Location 2 } \\
\text { (Workspace) }\end{array}$ & $\begin{array}{c}\text { Location 3 } \\
\text { (Workspace) }\end{array}$ \\
\hline $\begin{array}{c}\text { Location 4 } \\
\text { (Storage space) }\end{array}$ & $\begin{array}{c}\text { Location 5 } \\
\text { (Storage space) }\end{array}$ & $\begin{array}{c}\text { Location 6 } \\
\text { (Storage space) }\end{array}$ \\
\hline
\end{tabular}

Fig. 1. Layout of the facility for a small DSAP instance.

Table 2

Distances between locations

\begin{tabular}{|c|c|c|c|c|c|c|}
\hline & 1 & 2 & 3 & 4 & 5 & 6 \\
\hline 1 & - & 1 & 2 & 1 & 2 & 3 \\
\hline 2 & 1 & - & 1 & 2 & 1 & 2 \\
\hline 3 & 2 & 1 & - & 3 & 2 & 1 \\
\hline 4 & 1 & 2 & 3 & - & 1 & 2 \\
\hline 5 & 2 & 1 & 2 & 1 & - & 1 \\
\hline 6 & 3 & 2 & 1 & 2 & 1 & - \\
\hline
\end{tabular}

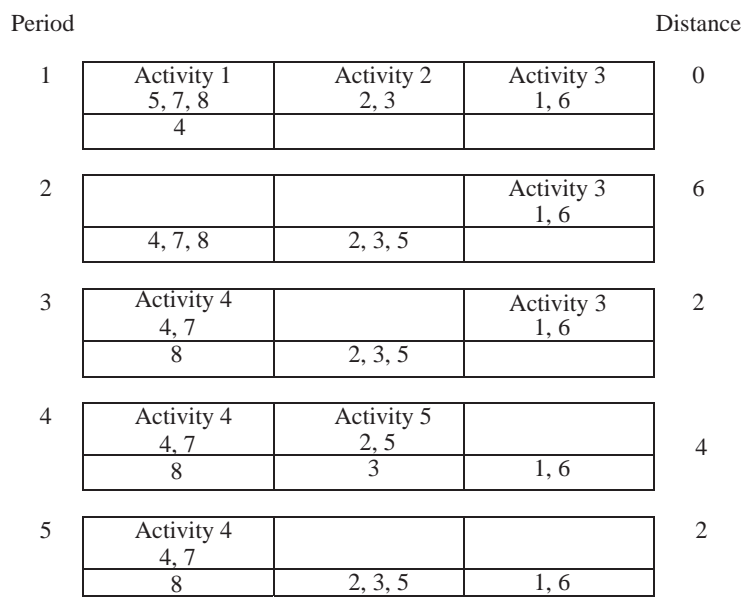

Fig. 2. A solution for the DSAP instance.

of 14 distance units (i.e., the total distance the resources travel is 14 distance units). In period 1, activities 1,2 , and 3 as well as their required resources are assigned to locations 1, 2, and 3, respectively, which are workspaces. Furthermore, the idle resource 4 is assigned to location 4 . Therefore, period 1 gives the initial assignment of resources to locations (work/storage spaces). To obtain the layout in period 2, the required resources for activities 1 and 2 in period 1 are moved to storage spaces at the beginning of period 2 (i.e., they become idle resources), since they are not required to perform an activity. More specifically, resources 2 and 3 are moved one distance unit from location 2 to location 5 (i.e., cost $=2$ ), and resource 5 is moved two distance units from location 1 to location 5 (i.e., cost $=2$ ). Finally, resources 7 and 8 are moved one distance unit from location 1 to location 4 (i.e., cost $=2$ ). Therefore, the total cost of the layout in period 2 is 6 distance units. In addition, the cost of the layouts in periods 3, 4, and 5 are 2, 4, and 2 distance units, respectively. Hence, the total distance the resources travel is 14 distance units. 
The assumptions for the DSAP are defined below:

(1) The locations of the workspaces and storage spaces (i.e., layout configuration) are known.

(2) The distances between locations are determined a priori.

(3) The resources required to perform each activity are known.

(4) The schedule of the activities is given.

(5) Only one activity can be performed in each workspace in a given period, and the workspace assigned to an activity is large enough to perform the activity and store its required resources.

(6) Each activity requires only one workspace and at least one resource.

(7) If an activity is performed in multiple periods, the activity is assigned to the same workspace each period it is performed.

(8) The capacities of the storage spaces are known.

(9) The objective is to minimize the total distance the resources travel throughout the duration of the project.

(10) If a resource is idle in consecutive periods, it is assigned to the same storage space location during those periods.

Assumptions (1)-(9) are the assumptions given in McKendall et al. [1]. However, assumption (10) was added, since it reduces the solution space by reducing the number of times an idle resource is moved during project implementation. This is illustrated using the DSAP solution in Fig. 2. For example, if assumption (10) is ignored and the capacity of location 4 is 4 resources, then resource 5 in periods 2 and 3 could have been assigned to locations 4 and 5, respectively, which gives the same total cost. However, the solution space increases, and the number of times that resource 5 is moved increase by one. For larger problems with many periods and locations, the increase could have been much larger, which could increase computation time. Furthermore, in reality, if the setup times required to prepare the resources to be moved are costly, then either minimizing the number of moves performed should be considered in the objective function or assumption (10) should be imposed.

\section{A tabu search heuristic for the DSAP}

Tabu search was first introduced by Glover [8]. Skorin-Kapov [9] was the first to present a tabu search heuristic for the QAP. Also, Skorin-Kapov [10], Chiang and Kouvelis [11], and Chiang and Chiang [12] presented tabu search heuristics for the QAP. In addition, Kaku and Mazzola [13] presented the only tabu search heuristic for the DFLP, known to the authors. Since the tabu search heuristic performed extremely well for the DFLP, which is similar to the DSAP, it is applied to solve the DSAP. Next, a tabu search (TS) heuristic is presented for the DSAP.

At an initial or current solution, the TS heuristic explores all the possible candidate moves in the neighborhood of the current solution using a pairwise exchange heuristic, and the best neighboring solution (or best move) is considered, with respect to the best objective function value, even if it results in a worse objective function value than the current solution (i.e., allows uphill moves to escape from getting trapped at a poor local optimum). To prevent cycling, the most recent moves are forbidden (or declared tabu) for a certain number of iterations. The number of iterations the move is tabu is called the tabu duration. The list of recent moves and its tabu duration is maintained in the tabu list. However, if a 
Table 3

Activity matrix for DSAP solution

\begin{tabular}{llll}
\hline & 1 & 2 & 3 \\
3 & & & 3 \\
& 4 & 5 & 3 \\
4 & 4 & & \\
& 4 & & \\
\end{tabular}

Table 4

Idle resource matrix for DSAP solution

\begin{tabular}{|c|c|c|c|c|c|c|c|c|}
\hline & 4 & & & & & & & \\
\hline & 4 & 7 & 8 & 2 & 3 & 5 & & \\
\hline \multirow[t]{3}{*}{$I=$} & & & 8 & 2 & 3 & 5 & & \\
\hline & & & 8 & & 3 & & 1 & 6 \\
\hline & & & 8 & 2 & 3 & 5 & 1 & 6 \\
\hline
\end{tabular}

move that gives the best solution ever is tabu, then the tabu restriction can be overridden, and the move is performed. The condition that allows the tabu restriction placed on a move to be overridden is called an aspiration criterion. Therefore, the best allowable move selected is either a tabu move that gives the best solution ever or the best move that is not classified as tabu (nontabu). The solution obtained after performing the best allowable move is defined as the current solution at the next iteration, and the process is repeated until a stopping criterion is met. At each iteration, the best solution found and its costs are saved and/or updated if necessary.

The initial solution is represented using three matrices: the activity matrix $A$, the idle resource matrix $\boldsymbol{I}$, and the dummy idle resource matrix $\boldsymbol{D}$. In Fig. 2, a solution to the DSAP example was given and was obtained by using a construction algorithm presented in this paper. The activity matrix $\boldsymbol{A}$ for the initial solution is given in Table 3 where the rows and the columns represent the sets of periods and workspaces, respectively. For example, activity 3 is assigned to location 3 in periods 1, 2, and 3 . The idle resource matrix $I$ for the initial solution is given in Table 4, where the rows represent the periods and the number of columns is based on the capacities of the storage spaces. In other words, the first-three, second-three, and third-three columns represent the available spaces in locations 4,5 , and 6 , respectively, since the capacity of the storage locations is three resources. For example, in periods 4 and 5, idle resources 1 and 6 are assigned to the first two spaces in storage location 6 . To obtain the dummy idle resource matrix $\boldsymbol{D}$, consider the idle resource matrix in Table 4. Start in period 1 and assign the first idle resource (resource 4 ) in consecutive periods it is idle to dummy idle resource 1. Since there are no more idle resources in period 1, go to period 2 and assign the first idle resource (resource 2) in consecutive periods it is idle to dummy idle resource 2 . Continue this process until all of the resources have been assigned to a dummy idle resource. See Table 5 for the resulting dummy idle resource matrix $\boldsymbol{D}$. In this example, resource 2 is associated with dummy idle resource 4 in periods 2 and 3 and with dummy idle resource 9 in period 5. Since the idle resources in consecutive periods are assigned to the same storage spaces, as defined in assumption (10), the dummy idle resource matrix structure is exploited such that a combination of moves can be defined by a single move (which reduces the solution space) and the definitions of the moves are 
Table 5

Dummy idle resource matrix

\begin{tabular}{llllllll}
\hline & 1 & & & & & \\
\\
$\boldsymbol{D}=$ & 2 & 3 & 4 & 5 & 6 & & \\
& & 3 & 4 & 5 & 6 & & \\
& & 3 & & 5 & & 7 & 8 \\
\hline
\end{tabular}

\begin{tabular}{|c|c|c|c|}
\hline \multirow[b]{2}{*}{ 1) } & \multicolumn{2}{|r|}{1} & 2 \\
\hline & $t=1$ & 2 & 1 \\
\hline & & 2 & 3 \\
\hline 2) & $t=1$ & 3 & 2 \\
\hline & $t=2$ & 3 & \\
\hline & $\mathrm{t}=3$ & 3 & \\
\hline
\end{tabular}

3)

\begin{tabular}{l|l|l|}
\multicolumn{1}{l|}{1} & \multicolumn{2}{c}{2} \\
\cline { 2 - 3 }$t=3$ & & 4 \\
\cline { 2 - 3 } & 5 & 4 \\
\cline { 2 - 3 } & & 4 \\
\cline { 2 - 3 } & &
\end{tabular}

4) $t=4$\begin{tabular}{|l|l|}
\hline & 3 \\
\hline & 5 \\
\hline
\end{tabular}
5)

\begin{tabular}{l|l|l|} 
& \multicolumn{1}{c}{1} & \multicolumn{1}{c}{3} \\
\cline { 2 - 3 }$t=1$ & 3 & 1 \\
\cline { 2 - 3 }$t=2$ & 3 & \\
\cline { 3 - 4 }$t=3$ & 3 & 4 \\
\cline { 3 - 4 }$t=4$ & & 4 \\
\hline & & 4 \\
\cline { 2 - 3 } & &
\end{tabular}

Fig. 3. All possible activity moves for the DSAP example.

simplified. More importantly, it simplifies the tabu list for keeping track of the most recent idle resource moves. This will be illustrated after defining an idle resource move.

Once the activity $(\boldsymbol{A})$, idle resource $(\boldsymbol{I})$, and dummy idle resource $(\boldsymbol{D})$ matrices have been defined, which represent the initial or current solution, all the solutions in the neighborhood of the current solution are obtained by considering all possible moves for the current solution. The increase or decrease in the transportation cost of the resources for each move is obtained, and the one that decreases/increases the transportation cost the most/least is considered. If this move is nontabu, the solution obtained from performing this move is defined as the current solution at the next iteration. If the move is tabu but gives the best solution ever, then the tabu restriction is overridden (aspiration criterion), and this move is selected and performed such that the corresponding neighboring solution is the current solution at the next iteration. However, if the move is tabu and does not satisfy the aspiration criterion, the next best move which is notabu is selected and performed such that the corresponding neighboring solution is the current solution at the next iteration.

There are two types of moves: activity and idle resource moves. When performing an activity move, it is important that assumption (7) is imposed to maintain feasibility. Therefore, the activity moves are defined as follows:

(1) interchange the locations (workspaces) of two or more activities in the periods the activities are performed;

(2) remove an activity from a workspace and assign it to an available (empty) workspace during the periods the activity is performed;

(3) a combination of both (1) and (2);

All the possible activity moves for the DSAP solution in Fig. 2 is given in Fig. 3, considering its activity matrix in Table 3. There are only five possible moves. Move 1 is an example of the first type of move where the locations of activities 1 and 2 in period 1 are interchanged. Move 4 is an example of the second 
(a)

\begin{tabular}{|l|l|l|l|l|l|l|l|l|}
\hline 1 & & & & & & & & \\
\hline 1 & 2 & 3 & 4 & 5 & 6 & & & \\
\hline & & 3 & 4 & 5 & 6 & & & \\
\hline & & 3 & & 5 & & 7 & 8 & \\
\hline & & 3 & 9 & 5 & 10 & 7 & 8 & \\
\hline
\end{tabular}

(b)

\begin{tabular}{|l|l|l|l|l|l|l|l|l|}
\hline 1 & & & & & & & & \\
\hline 1 & 2 & & 4 & 5 & 6 & 3 & & \\
\hline & & & 4 & 5 & 6 & 3 & & \\
\hline & & $\mathbf{7}$ & & 5 & & 3 & 8 & \\
\hline & & $\mathbf{7}$ & 9 & 5 & 10 & $\mathbf{3}$ & 8 & \\
\hline
\end{tabular}

Fig. 4. Dummy idle resource matrix (a) before move and (b) after move.

type of move where activity 5 is removed from location 2 and reassigned to location 3 in period 4 . Moves 2,3 , and 5 are examples of the third type of move.

When performing an idle resource move, it is important that assumption (10) is imposed, which reduces the solution space and the number of times idle resources are moved to/from locations during project duration. Therefore, the idle resource moves are defined below using the dummy idle resource matrix $\boldsymbol{D}$ :

(1) interchange the locations (storage spaces) of two or more idle resources assigned to different storage spaces in the consecutive periods they are idle;

(2) remove an idle resource from a storage location and assign it to an available (empty) storage space during the consecutive periods it is idle;

(3) a combination of both (1) and (2).

Considering the dummy idle resource matrix $\boldsymbol{D}$ in Table 5 (where storage locations 4, 5, and 6 have a capacity of three resources), interchanging the locations of dummy idle resources 3 in location 4 (column 3 ) and 5 in location 5 (column 5) in periods 2-5 is an example of the first type of move. An example of the second type of move is to remove dummy idle resource 3 in location 4 (column 3 ) and reassign it to the available space in location 6 (column 9), in periods 2-5. Removing dummy idle resource 3 in location 4 (column 3 ) in periods 2 and 3 and reassigning it to the available space in location 6 (column 7) as well as interchanging the locations of dummy idle resource 3 (i.e., idle resource 8) in location 4 (column 3) and dummy idle resource 7 (i.e., idle resource 1) in location 6 (column 7) in periods 4 and 5 is an example of the third type of move. See Fig. 4 for the dummy idle resource matrix $\boldsymbol{D}$ before and after the previous move is performed. It is important to note that the dummy idle resource matrix simplifies the definition of the idle resource moves by only considering moving an idle resource in consecutive periods in a single move (period). For instance, considering the idle resource matrix in Table 4, a candidate idle resource move is to remove idle resource 2 in location 5 (column 4) and assign it to an available space in location 6 (column 9) in periods 2 and 3. However, another separate candidate move is to remove idle resource 2 in location 5 (column 4) and assign it to an available space in location 6 (column 9) in period 5. Therefore, if the idle resource matrix $\boldsymbol{I}$ is used to define moves, instead of the dummy idle resource matrix $\boldsymbol{D}$, the list of recent moves would have to be maintained in a three-dimensional array considering the sets of idle resources, storage space locations, and periods. However, the dummy idle resource matrix $\boldsymbol{D}$ gives idle resource two different labels in different consecutive periods. In other words, idle resource 
Table 6

Activity tabu list for DSAP example

\begin{tabular}{rrrr}
\hline & 1 & 2 & 3 \\
$\boldsymbol{A}_{L}=$ & & & 15 \\
& 1 & 15 & 15 \\
3 & & \\
4 & & & \\
\hline
\end{tabular}

Table 7

Dummy resource tabu list for DSAP example

\begin{tabular}{|c|c|c|c|c|}
\hline & & 4 & 5 & 6 \\
\hline & 1 & & & \\
\hline & 2 & & & \\
\hline & 3 & & & 29 \\
\hline & 4 & & & \\
\hline \multirow[t]{6}{*}{$\boldsymbol{D}_{L}=$} & 5 & & & \\
\hline & 6 & & & \\
\hline & 7 & 29 & & 30 \\
\hline & 8 & 30 & & \\
\hline & 9 & & & \\
\hline & 10 & & & \\
\hline
\end{tabular}

2 in periods 2 and 3 is labeled as dummy idle resource 4 and labeled 9 in period 5 in Table 5 . Thus, the list of recent moves is maintained in a two-dimensional array where the rows and columns correspond to the set of dummy idle resources and storage space locations, respectively, which is illustrated below.

As discussed previously, the list of recent moves and its tabu duration are maintained and updated in the tabu list. Since we defined two types of moves (activity and idle resource moves), there are two tabu lists: activity $\left(\boldsymbol{A}_{L}\right)$ and dummy resource $\left(\boldsymbol{D}_{L}\right)$ lists. The activity tabu list $\boldsymbol{A}_{L}$ is a two-dimensional array where the rows and columns correspond to the sets of activities and workspace locations, respectively. In Table 6, the activity tabu list $\boldsymbol{A}_{L}$ for the DSAP example, after move 5 in Fig. 3 is performed, is given. Since move 5 was performed at this iteration (first iteration), the tabu list is updated such that the activity moves corresponding to moving activities 1,3 , and 4 to locations 3,1 , and 3, respectively, are tabu for 15 iterations (given the tabu duration is 15 iterations). At the next iteration, the tabu tenure of 15 is reduced to 14 for the three moves, and another move(s) will become tabu at this iteration where its tabu tenure will become 15. It is important to note, that any tabu move may be overridden if the move gives the best solution ever (i.e., aspiration criterion). The dummy resource tabu list $\boldsymbol{D}_{L}$ is also a two dimensional array such that the rows and columns correspond to the sets of dummy idle resources and storage space locations, respectively. After performing the move in Fig. $4 \mathrm{~b}$, the corresponding solution is the current solution at the next iteration (i.e., at iteration 2). If the moves which interchanges the locations of the dummy idle resources 7 and 8 is performed at the next iteration (iteration 2), then the dummy resource tabu list in Table 7 is obtained. Therefore, the idle resource moves corresponding to moving dummy idle resources 7 and 8 to locations 6 and 4, respectively, are tabu for 30 iterations (given the tabu duration is 
30 iterations). However, the idle resource moves corresponding to moving dummy idle resources 3 and 7 to locations 6 and 4, respectively, are tabu for 29 iterations. As previously stated, if the idle resource matrix was used to consider the idle resource moves (instead of the dummy idle resource matrix), then a three-dimensional resource tabu list would be needed to keep track of the most recent moves. That is, the sets of idle resources, storage space locations, and periods would be needed to keep track of the most recent moves. The tabu tenure for the activities is defined as three times the total number of activities, and the tabu tenure for the idle resources is defined as three times the total number of dummy idle resources. In both cases, the formulas were obtained experimentally.

An outline of the TS heuristic for the DSAP is given below.

Step 0: Generate several diverse initial solutions using the construction algorithms presented in the next section. Then perform the steps below for each of the initial solutions.

Step 1: For an initial solution obtained in the previous step, assign it to the current solution and obtain its cost. Set the current solution and its cost to the best solution ever and best cost ever, respectively. Obtain the activity $(\boldsymbol{A})$, idle resource $(\boldsymbol{I})$, and dummy idle resource $(\boldsymbol{D})$ matrices for the current solution. Also, initialize the activity $\left(\boldsymbol{A}_{L}\right)$ and dummy resource $\left(\boldsymbol{D}_{L}\right)$ tabu lists.

Step 2: Evaluate every possible activity and idle resource move for the current solution using the current activity $(\boldsymbol{A})$ and dummy idle resource $(\boldsymbol{D})$ matrices. Identify the best move that gives the lowest cost. If the best move is either classified as nontabu or tabu such that the aspiration criterion may be used to override the tabu status, then the move is performed, and the corresponding solution is defined as the current solution at the next iteration. Otherwise, if the best move is tabu such that the tabu restriction cannot be overridden with respect to the aspiration criterion, then select and perform the next best nontabu move. The corresponding solution is defined as the current solution at the next iteration.

Step 3: Considering the move performed in step 2, update the activity $(\boldsymbol{A})$ or dummy idle resource $(\boldsymbol{D})$ matrix and its corresponding tabu list $\left(\boldsymbol{A}_{L}, \boldsymbol{D}_{L}\right.$, respectively). Update the best solution ever and its cost, if necessary.

Step 4: The stopping criterion is defined as a certain number of iterations without improvement. The number obtained while performing several experimental runs is the product of the number of resources, number of activities, and number of periods. Therefore, if the stopping criterion has been met, terminate the heuristic. Otherwise, go to step 2.

\section{Construction algorithms for the DSAP}

The five construction algorithms presented in this section are used to generate initial solutions for the TS heuristic presented previously. The first algorithm is called the first assignment algorithm (FA), which assigns activities and idle resources to the first available space while maintaining feasibility. This algorithm is the same algorithm used in McKendall et al. [1] to generate initial solutions for their simulated annealing (SA) heuristics, except assumption (10) is considered here when constructing the solutions. The second algorithm is called the randomized clustering algorithm (RC) since it uses a clustering algorithm to assign activities to workspaces (similar to the algorithm proposed by Chan and Milner [14]) and the randomized storage policy to assign idle resources to storage spaces (similar to the randomized storage policy used to assign products to storage spaces in warehouses as in Tompkins et al. [15]). Also, the third and fourth algorithms are similar to the RC algorithm and are called the modified randomized clustering I and II algorithms, respectively. The fifth algorithm is similar to the FA algorithm. Therefore, 
this algorithm is called the modified first assignment algorithm (MFA). The details of each algorithm are discussed below.

As mentioned previously, the FA algorithm is similar to the method used to construct initial solutions in McKendall et al. [1]. However, solutions are constructed such that assumption (10) is imposed. The algorithm starts in the first period and assigns the first activity to the first workspace, the second activity to the second workspace, and so on. If an activity is performed in multiple periods, the activity is assigned to the same workspace in those periods (assumption (7)). Repeat the process for the remaining periods. The next step is to assign the idle resources to storage spaces. Start in the first period and assign the first $M$ (the capacity of the storage spaces) idle resources to the first storage space, the second $M$ idle resources to the second storage space, and so on, until all the idle resources in period 1 are assigned to storage spaces. If an idle resource is idle in consecutive periods, the resource is assigned to the same storage space in those periods (assumption (10)). Repeat the process for the remaining periods. As a result, this gives an initial solution for the DSAP.

To generate an initial solution for the DSAP using the RC algorithm, two matrices are needed to represent the problem. The first matrix is called the activity-resource matrix (ARM). The ARM is similar to the machine-part matrix used by the clustering algorithms in cellular manufacturing. The columns in the ARM represent the activities, and the rows represent the resources. An entry of " 1 " in position $(i, j)$ means that activity $j$ requires resource $i$ during its execution. The second matrix is called the schedule matrix (SM). The columns in the SM represent activities, and the rows represent periods. An entry of " 1 " in position $(i, j)$ of the SM means that activity $j$ is performed in period $i$. Once the ARM and SM are generated, the RC algorithm below can be used to obtain an initial solution for the DSAP. The first six steps are used to group the activities and their required resources into clusters. Step 7 assigns the clusters of activities to workspaces, and step 8 assigns the idle resources to storage spaces.

Step 1: Generate the ARM and SM, and obtain the rank index (RI) and interaction index (II) for each resource or row of the ARM.

- The RI is obtained by summing the number of entries in each row (for each resource).

- The II measures the number of resources that have bonds with each of the resources. In other words, this is the number of different resources used by activities that uses the resource under consideration.

Step 2: For the first or current cluster, the unassigned resources with the highest RI is moved to the first or current row, and ties are broken based on selecting the resource with the lowest II (if ties still exist, ties are broken randomly). More specifically, if no resources have been assigned, consider all unassigned resources. Otherwise, the unassigned resources with " $1 \mathrm{~s}$ " in the activities previously assigned to a cluster are considered for assignment in the next row, and the one with the highest RI and lowest II is selected. This row (resource) is defined as the current row (resource).

Step 3: Activities (columns) with "1s" in the current row are positioned together if there are no scheduling conflicts. A scheduling conflict exists when activities in the same cluster have ones in the same period (or row) in SM, since more than one activity cannot be assigned to the same workspace in the same period. If a scheduling conflict exists, assign the conflicting activity to the next cluster.

Step 4: If the number of resources (rows) assigned to the current cluster is less than the capacity of the corresponding storage space, then go to step 2.

Step 5: Close the current cluster. In other words, no more resources or activities can be assigned to the current cluster. 
Table 8

ARM (upper matrix) and SM (lower matrix) for DSAP example

\begin{tabular}{|c|c|c|c|c|c|c|c|}
\hline Res/Act & 1 & 2 & 3 & 4 & 5 & RI & II \\
\hline 1 & & & 1 & & & 1 & $1(6)$ \\
\hline 2 & & 1 & & & 1 & 2 & $2(3,5)$ \\
\hline 3 & & 1 & & & & 1 & $1(2)$ \\
\hline 4 & & & & 1 & & 1 & $1(7)$ \\
\hline 5 & 1 & & & & 1 & 2 & $3(2,7,8)$ \\
\hline 6 & & & 1 & & & 1 & $1(1)$ \\
\hline 7 & 1 & & & 1 & & 2 & $3(4,5,8)$ \\
\hline 8 & 1 & & & & & 1 & $2(5,7)$ \\
\hline
\end{tabular}

\begin{tabular}{lllll} 
Period & & 1 & 1 & \\
1 & 1 & 1 & & \\
2 & & 1 & 1 & \\
3 & & & 1 & 1 \\
4 & & & 1 & \\
5 & & & \\
\hline
\end{tabular}

Step 6: Repeat steps 2-5 until all resources and activities are associated with a cluster.

Step 7: The first cluster of activities is associated with the best-located workspace. The best-located workspace is defined as the workspace with the lowest cumulative distance with respect to the other spaces. In other words, the best-located workspace is the most central workspace. The second cluster of activities is assigned to the next best-located workspace. This operation is repeated until all the clusters of activities are associated with workspaces. Each cluster of activities is associated with the closest storage space.

Step 8: Allocate idle resources using the randomized storage policy. That is, idle resources are allocated to the closest storage spaces where the activities requiring the resources are assigned. Higher priority is given to the resources required sooner. Then calculate the cost of the solution. The cost is obtained by adding the distances the resources travel during the planning horizon.

To illustrate the RC algorithm, the DSAP example in Table 1 is considered. In step 1, the ARM and the SM are generated for the DSAP example and are given in Table 8. Also, the RI and the II are given in the table. The II is calculated by counting the number of resources interacting with each row. For example, resource 5 interacts with activities 1 and 5. In other words, activity 1 requires resources 5, 7, and 8, and activity 5 requires resources 2 and 5 . Therefore, resource 5 interacts with activities 1 and 5 , and it has a bond with resources 2, 7, and 8 (i.e., II =3). In step 2, resource 2 is moved to the first position, since it has the highest RI $(\mathrm{RI}=2)$ with the lowest II $(\mathrm{II}=2)$. Therefore, activities 2 and 5 are assigned to the first cluster, in step 3 . In step 4 , since resource 2 is the only resource assigned to the first cluster and the capacity of the corresponding storage space is three resources, step 2 is repeated. In step 2, only resources interacting with the current resource (resource 2) are considered for selection. That is, considering only resources $3(R I=1, I I=1)$ and $5(R I=2, I I=3)$, resource 5 is moved to the second row, since it has the highest RI. Notice that resource 5 is required by activities 1 and 5 where activity 5 is already assigned to the cluster. However, when step 3 is repeated, activity 1 cannot be assigned to the cluster, since there 
Table 9

Final ARM (upper matrix) and final SM (lower matrix) for DSAP example

\begin{tabular}{|c|c|c|c|c|c|c|c|}
\hline Res/Act & 2 & 5 & 1 & 4 & 3 & RI & II \\
\hline 2 & 1 & 1 & & & & 2 & 2 \\
\hline 5 & & 1 & 1 & & & 2 & 3 \\
\hline 7 & & & 1 & 1 & & 2 & 3 \\
\hline 3 & 1 & & & & & 1 & 1 \\
\hline 4 & & & & 1 & & 1 & 1 \\
\hline 8 & & & 1 & & & 1 & 2 \\
\hline 1 & & & & & 1 & 1 & 1 \\
\hline 6 & & & & & 1 & 1 & 1 \\
\hline \multicolumn{8}{|l|}{ Period } \\
\hline 1 & 1 & & 1 & & 1 & & \\
\hline 2 & & & & & 1 & & \\
\hline 3 & & & & 1 & 1 & & \\
\hline 4 & & 1 & & 1 & & & \\
\hline 5 & & & & 1 & & & \\
\hline
\end{tabular}

is a schedule conflict (i.e., a cluster cannot have two or more "1"s in the same row of the SM since only one activity can be assigned to a specific workspace at each period). Therefore, activity 1 is assigned to the next cluster. In step 4, since resources 2 and 5 are the only resources assigned to the first cluster, step 2 is repeated. In step 2, the resources interacting with resources 2 and 5 are considered for selection (i.e., resources 3 with RI $=1$ and II $=1,7$ with $\mathrm{RI}=2$ and $\mathrm{II}=3$, and 8 with $\mathrm{RI}=1$ and $\mathrm{II}=2$ ). As a result, resource 7 is moved to the third row, since it has the highest RI. In step 3, activity 4 is assigned to the next (second) cluster with activity 1 , since it conflicts with activity 5 in the SM. In step 4, since resources 2, 5, and 7 are assigned to the first cluster and the capacity of the storage spaces is 3 resources, then go to step 5. In step 5, the first cluster is completed, since the storage capacity has been met. In other words, resources 2, 5, and 7 are assigned to the first cluster, and more importantly, activities 2 and 5 are assigned to this cluster. The algorithm continues in this fashion. Since resources 3 and 4 has a tie for the lowest RI and II, either of them can be moved to the fourth position. As a result, resource 3 is chosen randomly. In summary, resources 3, 4, and 8 are assigned to the fourth, fifth, and sixth positions, respectively, which completes the second cluster. More importantly, activities 1 and 4 are assigned to the second cluster. Likewise, in the third cluster, resources 1 and 6 are assigned to the seventh and eighth positions, respectively, and activity 3 is assigned to the last column. Since all the activities and resources are associated with a cluster, the final ARM and SM is completed and given in Table 9. After generating the clusters, step 7 assigns the clusters of activities to workspaces. The first cluster (activities 2 and 5) is assigned to the most central workspace (location 2). The second (activities 1 and 4) and third (activity 3) clusters are assigned to locations 1 and 3, respectively. Fig. 5 shows the assignments of clusters to locations. Step 8 assigns the idle resources to storage spaces using the randomized storage policy, and the cost of the solution is obtained. The randomized storage policy assigns idle resources to the storage spaces closest to the workspaces they are assigned to, when performing activities. For instance, for periods 1 and 2 , idle resource 4 is assigned to location 4 , since it is required in location 1 at period 3. Similarly, 


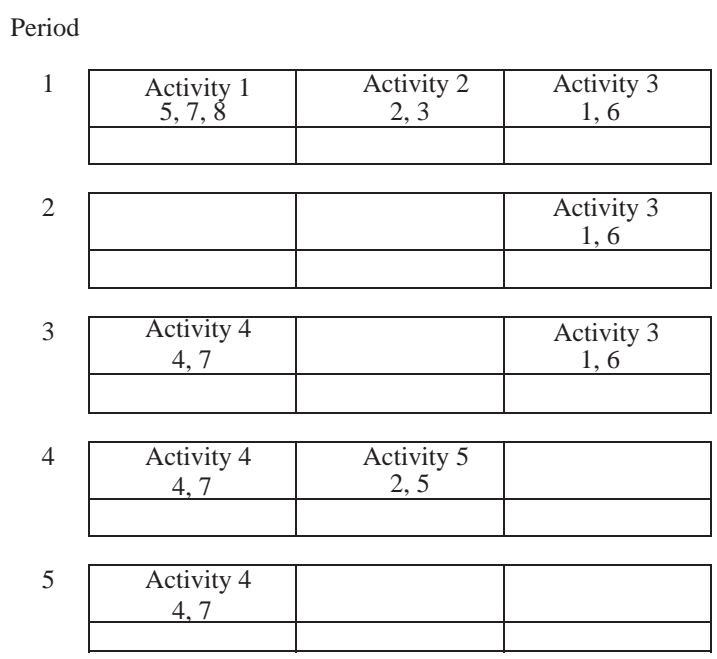

Fig. 5. Partial DSAP solution for the RC algorithm.

resource 2 is assigned to location 5 in periods 2 and 3, since it is used in location 2 at periods 1 and 4 . Since resource 3 is not needed to perform an activity after period 1, it is assigned to location 5 in periods 2, 3, 4, and 5. Continuing in this fashion, the solution in Fig. 2 is obtained, with a cost of 14 distance units.

The first modified RC (MRC I) algorithm uses the RC algorithm and the TS heuristic presented in the previous section. More specifically, the $\mathrm{RC}$ algorithm is used to obtain the assignment of activities to workspaces (partial DSAP solution). Recall, the partial solution for the DSAP example was given in Fig. 5. Notice that the cost of the partial solution is 1 distance unit. That is, it is the cost of moving resource 5 in period 1 from location 1 to location 2 in period 4 . Next, the partial initial solution is improved by applying the TS heuristic presented in Section 3. However, the tabu tenure is defined as the number of activities, and the stopping criterion is defined as $N$ iterations without improvement, where $N$ is the product of the number of activities and the number of workspaces. After improving the partial solution using the TS heuristic, the randomized storage policy is used to assign the idle resources to storage spaces, and the resulting solution is an initial solution for the TS heuristic.

The second modified RC (MRC II) algorithm is the same as the MRC I algorithm, except that steps 4 and 5 are ignored when forming the clusters. In other words, all the resources and activities are assigned to positions using steps 1-3 of the RC algorithm. However, the clusters of activities are determined based on scheduling conflicts. That is, the first $K_{1}$ activities without scheduling conflicts in the final ARM are assigned to the first cluster. The second $K_{2}$ activities without scheduling conflicts are assigned to the second cluster, and so on. After assigning the clusters of activities to workspaces as in step 7 of the RC algorithm, the assignment of activities to workspaces are improved using the TS heuristic as mentioned in the MRC I algorithm. Then the randomized storage policy is used to assign the idle resources to storage spaces as in step 8 of the RC algorithm. As a result, a different initial solution is obtained for the DSAP.

The last algorithm uses the FA algorithm to obtain the partial solution (i.e., the assignment of activities to workspaces), and the TS heuristic discussed previously is used to improve this solution. Then the 
randomized storage policy is used to assign the idle resources to storage spaces. This technique is called the modified FA algorithm. Therefore, the five construction algorithms presented in this section are used to obtain a diverse set of initial solutions for the TS heuristic presented in Section 3. Initially, a diversification strategy using frequency-based memory was implemented and compared to this strategy, but it was much less effective. Similar results were obtained by Kaku and Mazzola [13] for the DFLP.

\section{Computational results}

A set of test problems provided by McKendall et al. [1] were used to test the performances of the construction algorithms and the TS heuristic presented in this paper. The set consists of 96 test problems, which contain problems with 6,12,20, and 32 locations and 9, 15, 30, and 48 resources, respectively, each with 10,15 , and 20 periods. Half the locations are workspaces and the other half are storage spaces. Each of the storage spaces has a maximum capacity of three resources, and the number of required resources per activity varies between 1 and 3. Also, the number of activities ranges between 6 (for smaller problems) and 87 (for larger problems). The proposed heuristics were programmed using the Visual Basic programming language, and the set of test problems were solved on a Pentium IV 1.8 GHz PC. First, each test problem was solved using the construction algorithms, and the results are given under the "Initial" solutions column under each construction algorithms name in Tables 10,11,12, and 13. Next, the TS heuristic was used to improve the initial solutions, and the results are given under the "Final" solutions column, and the total time it took in minutes to obtain the initial and final solutions are given under the "Time" column. In the last three columns, the best solutions obtained using the SA heuristics presented in McKendall et al. [1] and its average computational times as well as the percent deviations the best TS heuristic solutions are away from the best SA heuristic solutions are given, respectively. The bold numbers give the best solution for each test problem, and the asterisks indicate that the solution obtained is optimal. The optimal solutions were obtained by McKendall et al. [1] for 25 of the test problems: for the first 24 test problems (P01-P24) and problem P27. Also, the italic numbers in the "Initial" columns give the best initial solution obtained for each test problem.

For the construction algorithms, the FA, RC, MFA, MRC I, and MRC II produced the optimal solution $2,4,9,10$, and eight times, respectively, for the 25 test problems in which the optimal solution is known. The FA, RC, MFA, MRC I, and MRC II algorithms produced the best initial solution 4, 17, 44, 51, and 48 times for the 96 test problems, respectively. Therefore, the MRC I construction algorithm outperformed the other algorithms with respect to solution quality. However, the initial solutions obtained for each test problem using each construction algorithm were obtained in less than $1 \mathrm{~min}$. After improving the initial solutions using the TS heuristic, the initial solutions obtained using the FA, RC, MFA, MRC I, and MRC II produced the optimal final solution 7, 16, 23, 21, and 20 times, respectively, for the 25 test problems in which the optimal solution is known. The FA, RC, MFA, MRC I, and MRC II with the TS heuristic obtained the best final solution 22, 33, 46, 46, and 46 times, respectively, for the 96 test problems. Therefore, the TS heuristic with the modified construction algorithms (MFA, MFC I, and MFC II) outperformed the TS heuristic using the other two construction algorithms (FA and RC), with respect to solution quality. More importantly, the computational times were much less. For instance, problem P96 required a total of $99.05,230.15,9.83,10.81$, and 9.75 min using the TS heuristic with the FA, RC, MFA, MRC I, and MRC II construction algorithms, respectively. In other words, it took almost $4 \mathrm{~h}$ to obtain a poor solution of 515 distance units using the TS heuristic with the RC algorithm and only approximately 
Table 10

Construction algorithms and TS heuristic results for problems with $N=6$

\begin{tabular}{|c|c|c|c|c|c|c|c|c|c|c|c|c|c|c|c|c|c|c|}
\hline \multirow[t]{2}{*}{$\mathbf{P b}$} & \multicolumn{3}{|l|}{ FA } & \multicolumn{3}{|l|}{$\mathbf{R C}$} & \multicolumn{3}{|l|}{ MFA } & \multicolumn{3}{|c|}{ MRC I } & \multicolumn{3}{|c|}{ MRC II } & \multicolumn{2}{|c|}{ BEST SA } & \multirow[t]{2}{*}{$\%$ Deviation } \\
\hline & Initial & Final & Time & Initial & Final & Time & Initial & Final & Time & Initial & Final & Time & Initial & Final & Time & Final & Time & \\
\hline P01 & 18 & $16^{*}$ & 0.02 & $16^{*}$ & $16^{*}$ & 0.02 & 18 & $16^{*}$ & 0.00 & $16^{*}$ & $16^{*}$ & 0.00 & 18 & $16^{*}$ & 0.00 & $16^{*}$ & 0.29 & $0 \%$ \\
\hline P02 & 28 & 26 & 0.02 & 29 & 26 & 0.02 & 28 & $25^{*}$ & 0.00 & 28 & $25^{*}$ & 0.00 & 28 & $25^{*}$ & 0.00 & $25^{*}$ & 0.32 & $0 \%$ \\
\hline P03 & $18^{*}$ & $18^{*}$ & 0.02 & $18^{*}$ & $18^{*}$ & 0.02 & $18^{*}$ & $18^{*}$ & 0.00 & $18^{*}$ & $18^{*}$ & 0.00 & $18^{*}$ & $18^{*}$ & 0.00 & $18^{*}$ & 0.27 & $0 \%$ \\
\hline P04 & 29 & $25^{*}$ & 0.02 & 26 & $25^{*}$ & 0.02 & 26 & $25^{*}$ & 0.00 & 26 & $25^{*}$ & 0.00 & 26 & $25^{*}$ & 0.00 & $25^{*}$ & 0.27 & $0 \%$ \\
\hline P05 & 18 & 17 & 0.02 & $16^{*}$ & $16^{*}$ & 0.02 & $16^{*}$ & $16^{*}$ & 0.00 & $16^{*}$ & $16^{*}$ & 0.00 & 18 & 17 & 0.00 & $16^{*}$ & 0.43 & $0 \%$ \\
\hline P06 & 34 & 28 & 0.02 & 31 & $27^{*}$ & 0.02 & 31 & $27^{*}$ & 0.00 & 31 & $27^{*}$ & 0.00 & 31 & $27^{*}$ & 0.00 & $27^{*}$ & 0.45 & $0 \%$ \\
\hline P07 & $16^{*}$ & $16^{*}$ & 0.02 & 17 & $16^{*}$ & 0.03 & $16^{*}$ & $16^{*}$ & 0.00 & $16^{*}$ & $16^{*}$ & 0.00 & $16^{*}$ & $16^{*}$ & 0.00 & $16^{*}$ & 0.49 & $0 \%$ \\
\hline P08 & 34 & $31^{*}$ & 0.02 & 33 & $31^{*}$ & 0.02 & 34 & $31^{*}$ & 0.00 & 34 & $31^{*}$ & 0.00 & 34 & $31^{*}$ & 0.00 & $31^{*}$ & 0.39 & $0 \%$ \\
\hline P09 & 30 & 26 & 0.06 & 26 & $25^{*}$ & 0.06 & $25^{*}$ & $25^{*}$ & 0.01 & $25^{*}$ & $25^{*}$ & 0.01 & $25^{*}$ & $25^{*}$ & 0.01 & $25^{*}$ & 0.48 & $0 \%$ \\
\hline P10 & 51 & 49 & 0.05 & 52 & 49 & 0.06 & 48 & $46^{*}$ & 0.01 & 48 & $46^{*}$ & 0.01 & 48 & $46^{*}$ & 0.01 & $46^{*}$ & 0.57 & $0 \%$ \\
\hline P11 & 41 & 33 & 0.06 & 40 & $32^{*}$ & 0.06 & $32 *$ & $32^{*}$ & 0.01 & $32^{*}$ & $32^{*}$ & 0.01 & $32^{*}$ & $32^{*}$ & 0.01 & $32^{*}$ & 0.81 & $0 \%$ \\
\hline P12 & 51 & 43 & 0.06 & 54 & $41^{*}$ & 0.06 & 43 & $41^{*}$ & 0.01 & 43 & $41^{*}$ & 0.01 & 43 & $41^{*}$ & 0.01 & $41^{*}$ & 0.59 & $0 \%$ \\
\hline P13 & 32 & 30 & 0.06 & 31 & 30 & 0.05 & $28^{*}$ & $28^{*}$ & 0.01 & 31 & 29 & 0.01 & 31 & 29 & 0.01 & $28^{*}$ & 0.59 & $0 \%$ \\
\hline P14 & 54 & 48 & 0.05 & 47 & $45^{*}$ & 0.05 & 48 & 46 & 0.01 & 48 & 46 & 0.01 & 48 & 46 & 0.01 & $45^{*}$ & 0.51 & $0 \%$ \\
\hline P15 & 38 & 36 & 0.06 & 40 & 37 & 0.10 & $35^{*}$ & $35^{*}$ & 0.01 & $35^{*}$ & $35^{*}$ & 0.01 & $35^{*}$ & $35^{*}$ & 0.01 & $35^{*}$ & 0.49 & $0 \%$ \\
\hline P16 & 58 & $49^{*}$ & 0.05 & 65 & $49^{*}$ & 0.05 & 52 & $49^{*}$ & 0.01 & 52 & $49^{*}$ & 0.01 & 52 & $49^{*}$ & 0.01 & $49^{*}$ & 0.51 & $0 \%$ \\
\hline P17 & 50 & 38 & 0.12 & $35^{*}$ & $35^{*}$ & 0.11 & $35^{*}$ & $35^{*}$ & 0.02 & $35^{*}$ & $35^{*}$ & 0.02 & $35^{*}$ & $35^{*}$ & 0.02 & $35^{*}$ & 1.32 & $0 \%$ \\
\hline P18 & 73 & 63 & 0.10 & 69 & 62 & 0.09 & 64 & $60^{*}$ & 0.02 & 65 & 61 & 0.02 & 65 & 61 & 0.02 & $60^{*}$ & 1.05 & $0 \%$ \\
\hline P19 & 60 & 52 & 0.11 & 56 & $46^{*}$ & 0.10 & 47 & $46^{*}$ & 0.02 & $46^{*}$ & $46^{*}$ & 0.02 & $46^{*}$ & $46^{*}$ & 0.02 & $46^{*}$ & 1.16 & $0 \%$ \\
\hline P20 & 74 & 62 & 0.10 & 79 & 62 & 0.08 & 63 & $60^{*}$ & 0.02 & 63 & $60^{*}$ & 0.02 & 63 & $60^{*}$ & 0.02 & $60^{*}$ & 0.99 & $0 \%$ \\
\hline P21 & 57 & 51 & 0.11 & 58 & 47 & 0.11 & 51 & 47 & 0.02 & 55 & 47 & 0.02 & 50 & 47 & 0.02 & $46^{*}$ & 1.01 & $2.17 \%$ \\
\hline P22 & 82 & 68 & 0.14 & 77 & 68 & 0.09 & 73 & $67^{*}$ & 0.02 & 73 & $67^{*}$ & 0.02 & 72 & $67^{*}$ & 0.02 & $67^{*}$ & 1.13 & $0 \%$ \\
\hline P23 & 59 & 59 & 0.11 & 64 & 59 & 0.19 & 57 & $55^{*}$ & 0.02 & 57 & $55^{*}$ & 0.02 & 57 & $55^{*}$ & 0.02 & $55^{*}$ & 1.03 & $0 \%$ \\
\hline P24 & 83 & $74^{*}$ & 0.11 & 78 & $74^{*}$ & 0.10 & 77 & $74^{*}$ & 0.02 & 77 & $74^{*}$ & 0.02 & 77 & $74^{*}$ & 0.02 & $74^{*}$ & 1.1 & $0 \%$ \\
\hline
\end{tabular}


Table 11

Construction algorithms and TS heuristic results for problems with $N=12$

\begin{tabular}{|c|c|c|c|c|c|c|c|c|c|c|c|c|c|c|c|c|c|c|}
\hline \multirow[t]{2}{*}{$\mathbf{P b}$} & \multicolumn{3}{|l|}{ FA } & \multicolumn{3}{|l|}{ RC } & \multicolumn{3}{|l|}{ MFA } & \multicolumn{3}{|c|}{ MRC I } & \multicolumn{3}{|c|}{ MRC II } & \multicolumn{2}{|c|}{ BEST SA } & \multirow[t]{2}{*}{$\%$ Deviation } \\
\hline & Initial & Final & Time & Initial & Final & Time & Initial & Final & Time & Initial & Final & Time & Initial & Final & Time & Final & Time & \\
\hline P25 & 47 & 31 & 0.49 & 33 & 33 & 0.39 & 47 & 36 & 0.03 & 33 & 33 & 0.03 & 42 & 31 & 0.03 & 34 & 2.39 & $-8.82 \%$ \\
\hline P26 & 67 & 45 & 0.47 & 49 & 45 & 0.41 & 52 & 48 & 0.04 & 49 & 45 & 0.03 & 48 & 45 & 0.03 & 47 & 2.44 & $-4.26 \%$ \\
\hline P27 & 50 & 44 & 0.41 & 48 & $4^{*}$ & 0.47 & $43^{*}$ & $4^{*}$ & 0.03 & $43^{*}$ & $4^{*}$ & 0.03 & $43^{*}$ & $43^{*}$ & 0.03 & $4^{*}$ & 2.43 & $0 \%$ \\
\hline P28 & 65 & 58 & 0.36 & 65 & 56 & 0.45 & 55 & 55 & 0.04 & 55 & 55 & 0.04 & 55 & 55 & 0.04 & 55 & 2.31 & $0 \%$ \\
\hline P29 & 34 & 29 & 0.50 & 34 & 29 & 0.44 & 34 & 29 & 0.08 & 34 & 29 & 0.04 & 36 & 30 & 0.04 & 29 & 2.66 & $0 \%$ \\
\hline P30 & 59 & 51 & 0.40 & 55 & 51 & 0.36 & 52 & 50 & 0.03 & 55 & 50 & 0.03 & 55 & 50 & 0.03 & 50 & 2.58 & $0 \%$ \\
\hline P31 & 52 & 42 & 0.43 & 50 & 43 & 0.42 & 45 & 43 & 0.02 & 45 & 43 & 0.02 & 42 & 42 & 0.02 & 42 & 2.74 & $0 \%$ \\
\hline P32 & 82 & 75 & 0.39 & 81 & 72 & 0.36 & 69 & 69 & 0.04 & 69 & 69 & 0.04 & 69 & 69 & 0.04 & 69 & 3.16 & $0 \%$ \\
\hline P33 & 75 & 56 & 0.99 & 64 & 60 & 1.36 & 58 & 56 & 0.08 & 60 & 57 & 0.08 & 59 & 54 & 0.08 & 59 & 4.98 & $-8.47 \%$ \\
\hline P34 & 96 & 74 & 0.97 & 98 & 76 & 0.95 & 80 & 74 & 0.08 & 79 & 75 & 0.08 & 82 & 76 & 0.08 & 79 & 5.23 & $-6.33 \%$ \\
\hline P35 & 85 & 77 & 1.57 & 93 & 76 & 1.38 & 73 & 73 & 0.08 & 74 & 74 & 0.10 & 74 & 74 & 0.10 & 73 & 4.39 & $0 \%$ \\
\hline P36 & 104 & 99 & 1.17 & 113 & 102 & 0.88 & 96 & 96 & 0.09 & 96 & 96 & 0.09 & 96 & 96 & 0.09 & 90 & 4.53 & $6.67 \%$ \\
\hline P37 & 77 & 48 & 1.05 & 50 & 49 & 1.14 & 62 & 52 & 0.12 & 53 & 52 & 0.09 & 59 & 52 & 0.09 & 54 & 7.54 & $-11.11 \%$ \\
\hline P38 & 102 & 83 & 1.27 & 99 & 85 & 0.93 & 91 & 87 & 0.08 & 89 & 82 & 0.08 & 91 & 87 & 0.08 & 83 & 8.76 & $-1.20 \%$ \\
\hline P39 & 85 & 71 & 1.18 & 91 & 72 & 1.29 & 69 & 68 & 0.10 & 69 & 68 & 0.09 & 72 & 68 & 0.09 & 71 & 6.6 & $-4.23 \%$ \\
\hline P40 & 126 & 112 & 0.98 & 120 & 114 & 0.93 & 108 & 108 & 0.09 & 108 & 108 & 0.08 & 108 & 108 & 0.09 & 108 & 6.68 & $0 \%$ \\
\hline P41 & 113 & 80 & 3.47 & 103 & 80 & 1.85 & 96 & 83 & 0.17 & 89 & 80 & 0.16 & 89 & 80 & 0.16 & 85 & 8.96 & $-6.25 \%$ \\
\hline P42 & 138 & 108 & 2.81 & 130 & 104 & 3.77 & 111 & 106 & 0.16 & 124 & 116 & 0.15 & 116 & 112 & 0.15 & 113 & 10.09 & $-7.96 \%$ \\
\hline P43 & 137 & 118 & 1.84 & 134 & 113 & 3.77 & 114 & 112 & 0.19 & 112 & 112 & 0.20 & 112 & 112 & 0.20 & 110 & 10.29 & $1.82 \%$ \\
\hline P44 & 172 & 143 & 1.66 & 183 & 152 & 1.76 & 140 & 140 & 0.18 & 144 & 144 & 0.18 & 140 & 140 & 0.18 & 140 & 8.72 & $0 \%$ \\
\hline P45 & 113 & 71 & 2.05 & 87 & 74 & 1.99 & 71 & 67 & 0.17 & 67 & 67 & 0.16 & 73 & 69 & 0.17 & 74 & 12.1 & $-9.46 \%$ \\
\hline P46 & 156 & 121 & 2.00 & 140 & 116 & 1.99 & 124 & 117 & 0.15 & 128 & 116 & 0.14 & 122 & 116 & 0.15 & 122 & 13.44 & $-4.92 \%$ \\
\hline P47 & 132 & 117 & 2.39 & 145 & 116 & 2.05 & 119 & 117 & 0.19 & 118 & 116 & 0.19 & 118 & 117 & 0.18 & 116 & 11.06 & \\
\hline P48 & 197 & 174 & 2.67 & 183 & 170 & 2.62 & 175 & 173 & 0.19 & 173 & 170 & 0.19 & 175 & 170 & 0.19 & 171 & 9.5 & $-0.58 \%$ \\
\hline
\end{tabular}


Table 12

Construction algorithms and TS heuristic results for problems with $N=20$

\begin{tabular}{|c|c|c|c|c|c|c|c|c|c|c|c|c|c|c|c|c|c|c|}
\hline \multirow[t]{2}{*}{$\mathbf{P b}$} & \multicolumn{3}{|l|}{ FA } & \multicolumn{3}{|l|}{ RC } & \multicolumn{3}{|l|}{ MFA } & \multicolumn{3}{|c|}{ MRC I } & \multicolumn{3}{|c|}{ MRC II } & \multicolumn{2}{|c|}{ BEST SA } & \multirow[t]{2}{*}{$\%$ Deviation } \\
\hline & Initial & Final & Time & Initial & Final & Time & Initial & Final & Time & Initial & Final & Time & Initial & Final & Time & Final & Time & \\
\hline P49 & 68 & 46 & 3.44 & 61 & 46 & 4.56 & 68 & 47 & 0.29 & 61 & 47 & 0.30 & 68 & 46 & 0.31 & 49 & 15.24 & $-6.12 \%$ \\
\hline P50 & 110 & 66 & 5.20 & 78 & 67 & 5.02 & 73 & 67 & 0.24 & 75 & 67 & 0.24 & 72 & 66 & 0.25 & 69 & 15.65 & $-4.35 \%$ \\
\hline P51 & 83 & 62 & 6.61 & 69 & 59 & 4.89 & 63 & 62 & 0.31 & 62 & 62 & 0.32 & 70 & 57 & 0.30 & 57 & 14.15 & $0 \%$ \\
\hline P52 & 147 & 106 & 2.97 & 121 & 106 & 2.89 & 98 & 98 & 0.27 & 100 & 100 & 0.36 & 98 & 98 & 0.28 & 98 & 13.65 & $0 \%$ \\
\hline P53 & 82 & 51 & 4.06 & 61 & 51 & 3.31 & 82 & 50 & 0.41 & 61 & 51 & 0.27 & 72 & 52 & 0.26 & 52 & 16.15 & $-3.85 \%$ \\
\hline P54 & 99 & 71 & 3.76 & 78 & 70 & 4.11 & 83 & 74 & 0.26 & 83 & 73 & 0.25 & 83 & 73 & 0.27 & 72 & 15.89 & $-2.78 \%$ \\
\hline P55 & 85 & 67 & 4.67 & 77 & 64 & 5.24 & 63 & 63 & 0.29 & 63 & 63 & 0.29 & 64 & 63 & 0.29 & 63 & 14.03 & $0 \%$ \\
\hline P56 & 116 & 108 & 3.02 & 123 & 99 & 4.44 & 98 & 98 & 0.26 & 99 & 99 & 0.26 & 99 & 99 & 0.26 & 98 & 13.77 & $0 \%$ \\
\hline P57 & 119 & 71 & 13.42 & 83 & 68 & 10.49 & 99 & 72 & 1.74 & 79 & 70 & 0.56 & 99 & 73 & 0.92 & 76 & 24.03 & $-10.53 \%$ \\
\hline P58 & 162 & 109 & 10.91 & 136 & 111 & 10.09 & 125 & 110 & 0.49 & 132 & 111 & 1.23 & 124 & 111 & 0.90 & 112 & 22.89 & $-2.68 \%$ \\
\hline P59 & 151 & 111 & 10.09 & 134 & 102 & 8.77 & 116 & 105 & 0.66 & 109 & 104 & 0.68 & 113 & 107 & 0.66 & 103 & 23.11 & $-0.97 \%$ \\
\hline P60 & 227 & 167 & 14.16 & 217 & 185 & 6.77 & 165 & 165 & 0.60 & 166 & 165 & 0.62 & 166 & 166 & 0.64 & 162 & 20.4 & $1.85 \%$ \\
\hline P61 & 147 & 85 & 9.67 & 89 & 82 & 8.41 & 112 & 88 & 0.72 & 87 & 82 & 0.56 & 100 & 88 & 0.51 & 84 & 24.64 & $-2.38 \%$ \\
\hline P62 & 195 & 131 & 7.96 & 151 & 130 & 8.18 & 165 & 139 & 0.55 & 146 & 135 & 0.51 & 147 & 131 & 0.51 & 137 & 22.11 & $-5.11 \%$ \\
\hline P63 & 184 & 129 & 15.57 & 171 & 134 & 10.03 & 127 & 125 & 0.65 & 132 & 128 & 0.64 & 130 & 127 & 0.66 & 127 & 23.34 & $-1.57 \%$ \\
\hline P64 & 259 & 191 & 16.07 & 251 & 210 & 9.34 & 202 & 190 & 0.61 & 198 & 190 & 0.61 & 197 & 191 & 0.62 & 190 & 21.93 & $0 \%$ \\
\hline P65 & 173 & 109 & 20.95 & 133 & 108 & 16.40 & 125 & 110 & 1.06 & 129 & 113 & 2.25 & 127 & 108 & 1.03 & 120 & 30.13 & $-10.00 \%$ \\
\hline P66 & 243 & 162 & 24.35 & 200 & 162 & 17.01 & 170 & 158 & 0.93 & 179 & 158 & 0.95 & 177 & 164 & 0.99 & 167 & 35.78 & $-5.39 \%$ \\
\hline P67 & 223 & 172 & 17.82 & 219 & 172 & 21.93 & 178 & 166 & 1.51 & 169 & 163 & 1.41 & 167 & 160 & 1.38 & 164 & 30.48 & $-2.44 \%$ \\
\hline P68 & 328 & 264 & 19.23 & 292 & 254 & 18.20 & 239 & 239 & 1.45 & 244 & 244 & 1.50 & 243 & 243 & 1.33 & 244 & 25.22 & $-2.05 \%$ \\
\hline P69 & 211 & 123 & 16.79 & 152 & 127 & 16.55 & 155 & 127 & 0.87 & 148 & 129 & 0.97 & 145 & 121 & 0.89 & 133 & 27.89 & $-9.02 \%$ \\
\hline P70 & 274 & 190 & 21.58 & 247 & 184 & 16.89 & 232 & 198 & 0.86 & 211 & 194 & 0.86 & 209 & 194 & 0.85 & 191 & 43.09 & $-3.66 \%$ \\
\hline P71 & 277 & 194 & 14.64 & 249 & 174 & 29.15 & 178 & 172 & 1.06 & 179 & 174 & 1.12 & 176 & 174 & 1.06 & 179 & 25.62 & $-3.91 \%$ \\
\hline P72 & 385 & 296 & 19.76 & 360 & 286 & 20.24 & 285 & 278 & 1.22 & 284 & 280 & 1.18 & 274 & 270 & 1.13 & 274 & 24.41 & $-1.46 \%$ \\
\hline
\end{tabular}


Table 13

Construction algorithms and TS heuristic results for problems with $N=32$

\begin{tabular}{|c|c|c|c|c|c|c|c|c|c|c|c|c|c|c|c|c|c|c|}
\hline \multirow[t]{2}{*}{$\mathbf{P b}$} & \multicolumn{3}{|l|}{ FA } & \multicolumn{3}{|l|}{ RC } & \multicolumn{3}{|l|}{ MFA } & \multicolumn{3}{|c|}{ MRC I } & \multicolumn{3}{|c|}{ MRC II } & \multicolumn{2}{|c|}{ BEST SA } & \multirow[t]{2}{*}{$\%$ Deviation } \\
\hline & Initial & Final & Time & Initial & Final & Time & Initial & Final & Time & Initial & Final & Time & Initial & Final & Time & Final & Time & \\
\hline P73 & 115 & 75 & 46.16 & 92 & 77 & 39.74 & 115 & 77 & 2.09 & 92 & 77 & 3.92 & 110 & 77 & 2.41 & 85 & 26.55 & $-11.76 \%$ \\
\hline P74 & 151 & 107 & 27.67 & 151 & 107 & 25.93 & 107 & 103 & 1.46 & 120 & 101 & 1.46 & 121 & 105 & 1.49 & 108 & 25.89 & $-6.48 \%$ \\
\hline P75 & 160 & 113 & 31.56 & 154 & 114 & 40.95 & 119 & 115 & 2.04 & 115 & 111 & 2.02 & 117 & 115 & 2.04 & 117 & 23.92 & $-5.13 \%$ \\
\hline P76 & 266 & 170 & 49.80 & 239 & 170 & 20.11 & 163 & 161 & 1.83 & 156 & 156 & 2.27 & 171 & 165 & 1.93 & 160 & 21.42 & $-2.50 \%$ \\
\hline P77 & 127 & 73 & 39.31 & 86 & 76 & 31.19 & 127 & 75 & 3.70 & 86 & 78 & 2.06 & 136 & 76 & 1.63 & 79 & 19.07 & $-7.59 \%$ \\
\hline P78 & 161 & 103 & 41.41 & 134 & 105 & 39.21 & 146 & 117 & 1.56 & 120 & 109 & 2.19 & 121 & 106 & 1.64 & 111 & 23.91 & $-7.21 \%$ \\
\hline P79 & 190 & 113 & 43.33 & 164 & 124 & 31.25 & 113 & 112 & 2.09 & 116 & 114 & 2.05 & 118 & 114 & 2.09 & 116 & 24.83 & $-3.45 \%$ \\
\hline P80 & 287 & 185 & 44.16 & 249 & 195 & 25.97 & 186 & 183 & 1.80 & 182 & 179 & 1.89 & 184 & 181 & 1.89 & 182 & 25.14 & $-1.65 \%$ \\
\hline P81 & 206 & 124 & 99.47 & 146 & 130 & 48.71 & 172 & 131 & 3.16 & 149 & 126 & 3.24 & 140 & 126 & 3.18 & 140 & 36.06 & $-11.43 \%$ \\
\hline P82 & 288 & 187 & 47.77 & 260 & 188 & 107.29 & 192 & 184 & 3.16 & 187 & 178 & 3.33 & 189 & 178 & 3.22 & 183 & 38.82 & $-2.73 \%$ \\
\hline P83 & 320 & 196 & 97.21 & 289 & 222 & 73.81 & 214 & 204 & 4.38 & 206 & 200 & 6.81 & 203 & 201 & 4.42 & 200 & 42.12 & $-2.00 \%$ \\
\hline P84 & 475 & 328 & 62.74 & 400 & 322 & 74.44 & 293 & 293 & 5.36 & 288 & 287 & 4.18 & 287 & 287 & 4.54 & 287 & 32.35 & $0 \%$ \\
\hline P85 & 230 & 134 & 86.94 & 151 & 136 & 56.09 & 152 & 136 & 3.07 & 151 & 131 & 3.10 & 156 & 139 & 3.12 & 144 & 39.59 & $-9.03 \%$ \\
\hline P86 & 284 & 206 & 85.43 & 287 & 195 & 103.38 & 206 & 207 & 3.05 & 238 & 206 & 3.23 & 245 & 216 & 6.10 & 209 & 52.19 & $-6.70 \%$ \\
\hline P87 & 348 & 226 & 54.22 & 306 & 212 & 103.12 & 200 & 195 & 4.44 & 207 & 201 & 4.39 & 207 & 216 & 6.36 & 205 & 34.44 & $-4.88 \%$ \\
\hline P88 & 484 & 369 & 65.19 & 466 & 346 & 73.68 & 325 & 318 & 4.27 & 310 & 306 & 4.42 & 311 & 306 & 4.14 & 302 & 33.78 & $1.32 \%$ \\
\hline P89 & 345 & 179 & 147.86 & 251 & 184 & 182.95 & 199 & 177 & 7.12 & 239 & 194 & 7.23 & 221 & 187 & 7.25 & 195 & 61.9 & $-9.23 \%$ \\
\hline P90 & 437 & 276 & 97.88 & 385 & 275 & 95.68 & 294 & 269 & 6.79 & 312 & 274 & 7.21 & 284 & 270 & 7.17 & 278 & 74.94 & $-3.24 \%$ \\
\hline P91 & 488 & 331 & 31.30 & 466 & 311 & 273.68 & 301 & 293 & 10.53 & 302 & 297 & 10.26 & 296 & 291 & 9.82 & 293 & 62.04 & $-0.68 \%$ \\
\hline P92 & 676 & 419 & 87.20 & 639 & 430 & 162.85 & 407 & 407 & 9.74 & 406 & 406 & 10.11 & 399 & 399 & 10.22 & 395 & 52.9 & $1.01 \%$ \\
\hline P93 & 320 & 197 & 30.92 & 266 & 197 & 127.87 & 223 & 195 & 8.07 & 235 & 194 & 8.26 & 255 & 204 & 7.03 & 211 & 76.5 & $-8.06 \%$ \\
\hline P94 & 416 & 306 & 104.61 & 388 & 284 & 182.18 & 359 & 302 & 7.51 & 344 & 300 & 7.47 & 332 & 296 & 14.26 & 298 & 65.38 & $-4.70 \%$ \\
\hline P95 & 544 & 350 & 113.23 & 523 & 332 & 196.41 & 349 & 329 & 9.48 & 327 & 319 & 11.15 & 327 & 318 & 10.10 & 332 & 60.06 & $-4.22 \%$ \\
\hline P96 & 736 & 571 & 99.05 & 746 & 515 & 230.15 & 498 & 492 & 9.83 & 502 & 500 & 10.81 & 476 & 466 & 9.75 & 485 & 52.55 & $-3.92 \%$ \\
\hline
\end{tabular}


10 min to obtain the best solution of 466 distance units using the TS heuristic with the MRC II algorithm. As with other similar TS heuristics presented in the literature, the performance of the TS heuristic often depends on the quality of the initial solutions.

Although the TS heuristic produced the optimal solution for 24 of the 25 problems for which the optimal solution is known, the SA heuristics produced the optimal solutions for all 25 problems. Out of the 96 test problems, the SA heuristics and the TS heuristic both produced the best solution for $39(40.63 \%)$ of the problems, and the SA heuristics outperformed the TS heuristic for only $6(6.25 \%)$ of the test problems. However, the TS heuristic outperformed the SA heuristics for $51(53.13 \%)$ of the test problems. Out of the 51 test problems, $15(29.41 \%)$ of the solutions for the TS heuristic were within $3 \%$ below the best-found solution for the SA heuristics. Also, 13 (25.5\%), 18 (35.29\%), and 5 (9.8\%) of the solutions were within $3-5 \%, 5-10 \%$, and $10-12 \%$, respectively. It is important to note that the computational time given for the SA heuristics is the average time for a total of 18 runs ( 9 runs for each SA heuristic). For instance, the best solution of 485 distance units for test problem P96 was obtained in 18 runs using the SA heuristics such that each run required an average of $52.55 \mathrm{~min}$. For the best solution of 466 distance units for test problem P96 was obtained in 5 runs (i.e., for each initial solution obtained by each construction algorithm) using the TS heuristic such that each run required an average of $71.92 \mathrm{~min}$. However, the best solution of 466 distance units was obtained in three runs by the TS heuristic with the modified algorithms such that each run required an average of $10.13 \mathrm{~min}$. Therefore, the TS heuristic clearly outperformed the SA heuristics with respect to solution quality. In addition, the TS heuristic with the modified construction algorithms produced very good solutions with much less computational time.

As in McKendall et al. [1], the problems are listed such that the first, second, third, and fourth problems in each set of four problems (separated by bold lines) are categorized as hardest, hard, easy, and easiest problems, respectively. The authors define the harder problems as the problems where the average number of resources per activity and workspace utilization are relatively high (i.e., average number of idle resources is relatively high), and the easier problems are the problems where the average number of resources per activity and workspace utilization are relatively low (i.e., average number of idle resources is relatively low). By observing the percent deviations, the results clearly show that the SA heuristics perform worst for the harder problems than for the easier problems. In other words, the performances of the SA heuristics deteriorate as the problem size increases. More specifically, the performances of the SA heuristics deteriorate when solving harder problems (i.e., when the average number of idle resources is relatively high and the solution space increases).

The DSAP is the combination of the permutation problem of assigning activities to workspaces as well as the partitioning problem of partitioning idle resources to sets and then assigning these sets to locations (permutation problem). Since the tabu search heuristics often perform better than the simulated annealing heuristics available in the literature for permutation and partitioning problems, it is not surprising that the TS heuristic presented in this paper outperformed the SA heuristics presented in McKendall et al. [1] with respect to solution quality and computation time (specifically for the better construction algorithms). As in the literature, the solution quality and computational time usually depend on the quality of the initial solution for the tabu search heuristic, as discussed previously. Although the quality of the initial solutions for the SA heuristics does not determine the performance of the heuristic, the stochastic nature of the SA heuristics is the major drawback, since it becomes extremely difficult to effectively explore the solution space when the solution space is extremely large. This was observed when solving the harder problems, as discussed previously. 


\section{Conclusions}

In this paper, five construction algorithms and a TS heuristic were presented for the DSAP. The first assignment (FA) algorithm assigns activities and idle resources to the first available work/storage spaces while maintaining feasibility. The randomized clustering (RC) algorithm use a clustering algorithm to assign activities to workspaces and uses the randomized storage policy to assign idle resources to storage spaces. These algorithms were modified such that they were used to obtain the assignments of activities to workspaces (partial solutions). Then the TS heuristic was used to improve the partial solutions, and the randomized storage policy was used to assign the idle resources to storage spaces. The modified heuristics for the FA and RC algorithms are called the modified FA (MFA) and modified RC (MRC I) algorithms. The last algorithm called MRC II is exactly like MRC I, except that steps 4 and 5 in the $\mathrm{RC}$ algorithm is omitted. Of the five construction algorithms, the modified algorithms (MFA, MRC I, and MRC II) outperformed the first two algorithms (FA and RC) with respect to solution quality. Also, the TS heuristic with the modified algorithms clearly outperformed the TS heuristic with the first two construction algorithms with respect to both solution quality and computational time. More importantly, the TS heuristic presented in this paper clearly outperformed the SA heuristics presented in the literature for the DSAP with respect to solution quality and computational time. Although the TS heuristic presented in this paper performed extremely well, TS heuristics which consider dynamic tabu list, frequency-based memory, etc. are areas for further consideration.

\section{Acknowledgements}

This research was funded in part by NASA West Virginia EPSCoR Program.

\section{References}

[1] McKendall AR, Noble JS, Klein CM. Simulated annealing heuristics for managing resources during planned outages at electric power plants. Computers \& Operations Research 2005;32(1):107-25.

[2] Gharbi A, Pellerin R, Villeneuve L. A new constraint-based approach for overhaul project scheduling with work space constraints. International Journal of Industrial Engineering 1999;6(2):123-31.

[3] Zouein PP, Tommelein ID. Dynamic layout planning using a hybrid incremental solution method. Journal of Construction Engineering \& Management 1999;125(6):400-8.

[4] Rosenblatt MJ. The dynamics of plant layout. Management Science 1986;32(1):76-86.

[5] Koopmans T, Beckmann M. Assignment problems and the location of economic activities. Econometrica 1957;25:53-76.

[6] Balakrishnan J, Cheng $\mathrm{CH}$. Genetic search and the dynamic layout problem. Computers \& Operations Research 2000;27(6):587-93.

[7] Sahni S, Gonzalez T. P-complete approximation problems. Journal of the Association of Computer Machinery 1976;23:555 -65 .

[8] Glover F. Future paths for integer programming and links to artificial intelligence. Computers \& Operations Research 1986;1(3):533-49.

[9] Skorin-Kapov J. Tabu search applied to the quadratic assignment problem. ORSA J on Computing 1990;2:33-45.

[10] Skorin-Kapov J. Extensions of a tabu search adaptation to the quadratic assignment problem. Computers \& Operations Research 1994;21(8):855-65.

[11] Chiang W-C, Kouvelis P. An improved tabu search heuristic for solving facility layout design problems. International Journal of Production Research 1996;34(9):2565-85. 
[12] Chiang W-C, Chiang C. Intelligent local search strategies for solving facility layout problems with the quadratic assignment problem formulation. European Journal of Operations Research 1998;106:457-88.

[13] Kaku BK, Mazzola JB. A tabu-search heuristic for the dynamic plant layout problem. INFORMS Journal on Computing 1997;9(4):374-84.

[14] Chan HM, Milner DA. Direct clustering algorithm for group formation in cellular manufacture. Journal of Manufacturing Systems 1982;1:65-75.

[15] Tompkins JA, White JA, Bozer YA, Frazelle EH, Tanchoco JMA, Trevino J. Facilities planning. New York: Wiley, 1996. p. $418-34$. 